\title{
Life-threatening manifestation of cutaneous leukocytoclastic vasculitis
}

\author{
Marta Runowska, Dominik Majewski, Mariusz Puszczewicz
}

Department of Rheumatology and Internal Diseases, Poznan University of Medical Sciences, Poznan, Poland

Adv Dermatol Allergol 2021; XXXVIII (2): 335-337

DOI: https://doi.org/10.5114/ada.2021.106214

Cutaneous leukocytoclastic vasculitis (LCV) is a form of small-vessel vasculitis with a predilection for skin involvement. In about half of the cases the condition has an idiopathic origin, whereas the remaining cases develop secondarily to various causes, such as infections, connective tissue diseases, malignancies, and drugs [1]. Although LCV is generally considered as self-limiting and has a good overall prognosis [2], several studies have described less favourable, chronic, and/or recurrent course of the disease $[3,4]$. Hence, therapeutic options depend on the severity of disease, and range from antihistamines, topical and/or oral corticosteroids (CS), to immunosuppressive drugs $[4,5]$. Herein, we report the case of a 46-year-old man with a history of multiple, deep skin ulcerations over the trunk and extremities, later confirmed as LCV. The patient had been treated with a combination of systemic CS and infusions of intravenous immunoglobulins (IVIG) within the 29-month period of followup, which gradually lead to significant, although to date not complete, reduction of skin lesions, and remarkably improved the quality of the patient's life.

A 46-year-old Caucasian male with no significant previous medical history was admitted to the Rheumatology Department in December 2016, presenting with extensive skin ulcerations of the trunk and the extremities in the course of newly diagnosed LCV. The first symptoms of the disease developed about 6 months earlier and included recurrent swelling of the right leg, accompanied by purpuric rash on the lower extremities and subsequent formation of non-healing skin ulcerations. The patient's history of constitutional symptoms, preceding infection, and drug intake was negative. The laboratory results revealed raised inflammatory markers (erythrocyte sedimentation rate (ESR) $-52 \mathrm{~mm} / \mathrm{h}$, C-reactive protein (CRP) $-99.5 \mathrm{mg} / \mathrm{l})$, thrombocytosis, anaemia of chronic diseases (normocytic anaemia with low serum iron level, decreased TIBC, increased concentration of serum ferritin, normal RDW, and normal reticulocyte count), and decreased serum albumin and proteinuria. The serological findings, including antineutrophil cytoplasmic antibodies (ANCA), antinuclear antibodies (ANA), antiphospholipid antibodies (anticardiolipin and antiß2GP1 antibodies), were negative. Physical examination revealed numerous, extensive skin ulcerations with focal necrosis over the trunk, and the upper and lower extremities, including the hands and feet.

Intravenous CS and empiric antibiotic therapy was initiated; however, after transient clinical and laboratory improvement, the areas of ulcerations and necrosis became more extensive, requiring surgical debridement. The patient underwent oral sanation to eliminate the potential source of infection. Doppler ultrasonography of the lower limbs excluded the proximal venous thrombosis but failed to evaluate the distal veins because of the swelling and ulcerations of the calves. Meanwhile, the patient's general condition deteriorated, and he presented with neurological symptoms, including transient confusion and myoclonus. The magnetic resonance imaging (MRI) of the head showed two 3-5 $\mathrm{mm}$ ischaemic foci in the frontal lobes. Electroencephalography (EEG) did not record epileptiform discharges, while the attempts to perform lumbar puncture did not succeed. The histopathology from the skin biopsy revealed neutrophilic infiltration with leukocytoclasia and fibrinoid necrosis of the small vessels, features characteristic for LCV.

A differential diagnosis of potential underlying causes of LCV was performed. Because the patient presented with some systemic and organ-specific symptoms, ANCA-associated vasculitis (AAV) was seriously considered as a possible aetiological factor; however, at that moment, he did not fulfil the diagnostic criteria for AAV, and ANCA were negative. The patient was also screened

Address for correspondence: Marta Runowska MD, Department of Rheumatology and Internal Diseases, Poznan University of Medical Sciences, 135/147 28 Czerwca 1956r. St, 61-546 Poznan, Poland, phone: +48 6183103 17, e-mail: marta.runowska1@gmail Received: 27.07.2019, accepted: 16.09.2019. 

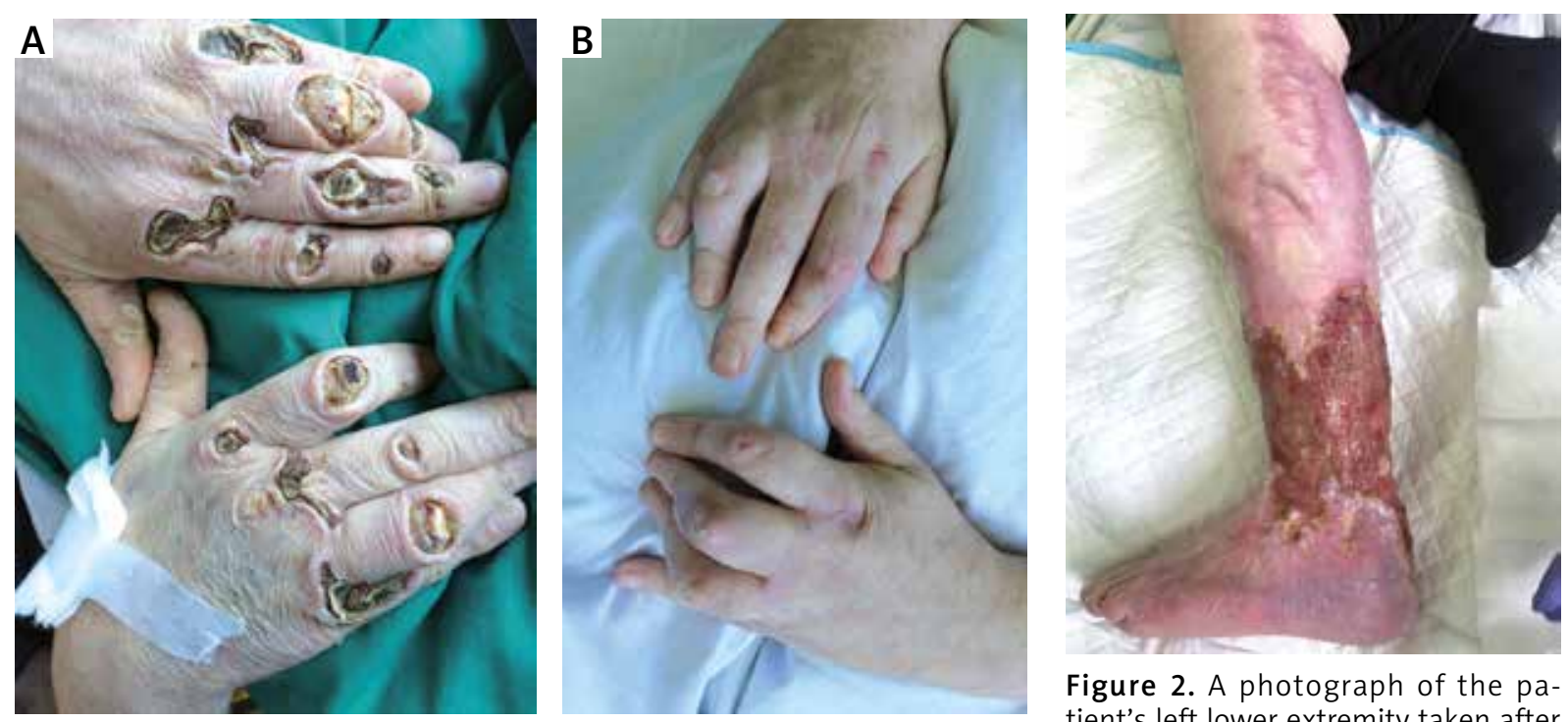

Figure 2. A photograph of the patient's left lower extremity taken after 29 months of follow-up, showing ulcer-

Figure 1. Photographs of the patient's hands taken at the first admission to the hospital, with deep ulcerations over the joints, revealing the surface of the bones (A), and after 10 months of follow-up (B), showing full resolution of ulcerations, with the persistence of joint contractures the thigh, after healed ulceration

for malignancies, and diagnostic tests such as CT of the chest and abdomen, gastroscopy, and tumour markers were carried out. Unfortunately, due to deterioration of the patient's general condition, a colonoscopy was not performed. Other secondary forms of vasculitis, such as cryoglobulinaemia, vasculitis associated with systemic diseases (e.g. SLE, RA, sarcoidosis), monoclonal gammopathies, and infections (including hepatitis B and C virus-associated vasculitis), were excluded.

The patient was treated with intravenous methylprednisolone pulses followed by oral CS, with an initial dose, in conversion to prednisone, of $0.8 \mathrm{mg} / \mathrm{kg} / \mathrm{day}$. Subsequently, cyclophosphamide therapy was initiated, and a course of plasmapheresis was performed, concurrent with therapeutic-dose heparin and broad-spectrum antibiotic therapy; however, no significant improvement was observed. Due to the deepening anaemia, the patient eventually required a blood transfusion. Eventually, IVIG therapy was initiated, at a dose of $30 \mathrm{~g}$ per infusion.

Since the first admission, the patient was hospitalized at the Rheumatology Department at regular 3-4-month intervals, with continuation of IVIG infusions and CS therapy. Simultaneously, surgical debridement of the ulcerations and treatment with silver-coated dressings were continued. As a result, gradual improvement of the patient's general condition and gradual healing of the ulcerations was observed, allowing reduction of the oral methylprednisolone dose over time. The improvement of laboratory results was also observed, including normalization of anaemia and proteinuria, and a decrease of inflammatory marker levels. Due to the prolonged immobilization, the patient initially developed significant muscular weakness and secondary joint con- tractures. After long-term rehabilitation, he is currently walking with support of crutches; however, the contractures of the hands persist.

During the last follow-up, the physical examination of the patient revealed extensive scars of healed ulcers on the trunk, hands, and lower extremities, and persisting ulceration in the area of the left shin and heel. A photograph of patient's skin lesions from the disease onset is presented in Figure $1 \mathrm{~A}$, and photographs of the lesions after 10 and 29 months of treatment are presented in Figures $1 \mathrm{~B}$ and 2 .

LCV, according to CHCC 2012 [6] is classified as "single organ vasculitis" (SOV), an entity encompassing vasculitides of any size of vessel localized in a single organ that has no features indicative for a limited expression of systemic vasculitis. However, some patients who were originally diagnosed as having SOV will develop additional disease manifestations that require redefining the case as one of systemic vasculitides. Therefore, due to its rarity, variable clinical picture, diversity of possible aetiological factors, and thus the need for thorough differential diagnosis performance, LCV can be challenging in both diagnostic and therapeutic aspects for clinicians of different specialities. In the presented case, some of the systemic and organ-specific symptoms, such as anaemia, proteinuria, and neurological symptoms, were initially observed, which was suggestive of the underlying systemic vasculitis. Although our patient did not meet the classification criteria of any of the systemic vasculitides, we cannot definitely exclude their limited expression, even in the absence of ANCA. Hence, long-term observation for new organ involvement, even after complete healing of skin lesions, is strongly indicated in this case. 
The most common clinical presentation of LCV is palpable purpura, typically localized on the lower extremities $[4,7,8]$. However, the range of other possible skin manifestations is wide and includes urticaria, bullae, nodules, vesicles, and petechiae. The symptoms presented by our patient, i.e. extensive skin ulcerations with necrosis, are not the most frequent clinical manifestations of the disease - ulcers are described in about 9-25\% of such cases $[4,7,8]$. Hence, especially in doubtful cases, a skin biopsy is recommended, to confirm the vasculitis as an underlying cause of the cutaneous lesions [5]. It should be noted, however, that once the leukocytoclastic vasculitis is identified by the biopsy, it is not possible to distinguish between its primary and secondary forms only on the basis of the histopathology findings.

With regards to the management, no randomized studies comparing the effectiveness of different drugs in LCV are available. However, it seems reasonable that the treatment strategy is determined by the aetiology, the severity of symptoms, and the extent of cutaneous involvement [5]. Bed rest, elevation of the lower extremities, nonsteroidal anti-inflammatory drugs (NSAIDs), analgesics, and antihistamines are usually recommended as the symptomatic treatment for complaints such as pruritus or burning; however, none of them significantly modifies the course of the disease or prevents recurrences [2]. In extensive, progressive, and/or recurrent manifestations, more aggressive treatment is required, including systemic CS and/or steroid-sparing agents such as colchicine, dapsone, azathioprine, hydroxychloroquine, or cyclophosphamide [4, 5, 9]. In the presented case, systemic CS monotherapy, cyclophosphamide, and plasmapheresis were ineffective, and therefore treatment with IVIG parallel to CS was initiated. After 29 months of follow-up, significant improvement of the patient's general condition and healing of most of the skin lesions was achieved, as a result of both systemic and surgical treatment combined with rehabilitation.

In conclusion, the presented case proves that despite overall good prognosis and a tendency for self-limitation, even skin-limited vasculitis may sometimes take a severe, disabling, or even life-threatening course, making its management a challenge.

\section{Conflict of conflict}

The authors declare no conflict of interest.

\section{References}

1. Ekenstam E, Callen J. Cutaneous leukocytoclastic vasculitis. Clinical and laboratory features of 82 patients seen in private practice. Arch Dermatol 1984; 120: 484-9.

2. Russell JP, Gibson LE. Primary cutaneous small vessel vasculitis: approach to diagnosis and treatment. Int I Dermatol 2006; 45: 3-13.
3. Sais G, Vidaller A, Jucglà A, et al. Prognostic factors in leukocytoclastic vasculitis. Arch Dermatol 1998; 134: 309-15.

4. Bouiller K, Audia S, Devilliers H, et al. Etiologies and prognostic factors of leukocytoclastic vasculitis with skin involvement: a retrospective study in 112 patients. Medicine 2016; 95: e4238.

5. Gota CE, Calabrese LH. Diagnosis and treatment of cutaneous leukocytoclastic vasculitis. Int J Clin Rheumatol 2013; 8: 49-60.

6. Jennette JC, Falk RJ, Bacon PA, et al. 2012 Revised International Chapel Hill Consensus Conference Nomenclature of Vasculitides. Arthritis Rheum 2013; 65: 1-11.

7. Arora A, Wetter DA, Gonzalez-Santiago TM, et al. Incidence of leukocytoclastic vasculitis, 1996-2010: a population-based study in Olmsted County, Minnesota. Mayo Clin Proc 2014; 89: 1515-24.

8. Cakiter A, Kucuk O, Ozkaya D, et al. Demographic characteristics, aetiology, and assessment of treatment options in leukocytoclastic vasculitis. Adv Dermatol Allergol 2017; 34: 104-9.

9. Einhor J, Levis JT. Dermatologic diagnosis: leukocytoclastic vasculitis. Perm J 2015; 19: 77-8. 